Article

\title{
An Experimental Approach for the Direct Measurement of Temperatures in the Vicinity of the Keyhole Front Wall during Deep-Penetration Laser Welding
}

\author{
Ronald Pordzik * and Peer Woizeschke [ \\ BIAS-Bremer Institut für angewandte Strahltechnik GmbH, Klagenfurter Str. 5, 28359 Bremen, Germany; \\ woizeschke@bias.de \\ * Correspondence: pordzik@bias.de; Tel.: +49-421-218-58078
}

Received: 8 May 2020; Accepted: 4 June 2020; Published: 6 June 2020

\begin{abstract}
The formation of defects such as pores during deep-penetration laser welding processes is governed by the melt pool dynamics and the stability of the vapor capillary, also referred to as the keyhole. In order to gain an insight into the dynamics of the keyhole, the temperature in the transition region from the liquid to the gaseous phase, i.e., near the keyhole wall, is a physical value of fundamental importance. In this paper, a novel method is presented for directly measuring temperatures in the close vicinity of the keyhole front wall during deep-penetration laser welding. The weld samples consist of pure aluminum with a boiling point of $2743 \mathrm{~K}$. The measurement is performed using high-speed pyrometry with a refractory tantalum probe capable of detecting temperatures that significantly exceed the boiling point of the sample material. Temperature curves are recorded from the beginning of the welding process until the moment the probe is finally destroyed through direct laser-tantalum interaction. With an effective spatial resolution up to $0.3 \mu \mathrm{m}$ in the welding direction, a detailed investigation into the temperature ranging from the prerunning melt pool front to the keyhole center is possible, exhibiting temperatures of up to $3300 \mathrm{~K}$ in the vicinity of the keyhole front wall.
\end{abstract}

Keywords: deep-penetration laser welding; keyhole behavior; keyhole dynamics; keyhole wall temperature; pyrometry; Knudsen layer

\section{Introduction}

Deep-penetration laser welding is a well-established yet still promising joining technology with a broad field of applications in the automotive, aerospace, and many other industries of great economic significance. Allowing for high-aspect ratios, it has proven to be an ideal method for generating deep and narrow weld seams at comparatively low energy inputs [1]. High welding velocities and low thermal distortion of the joined parts qualify this technology for applications in manufacturing processes, e.g., for automated welding tasks or complex welding contours [2]. At a certain material-dependent intensity threshold of the laser beam, the material evaporation induces a recoil pressure onto the molten material, allowing the laser to penetrate the formerly flat workpiece surface [3]. Thereby, a long and narrow vapor capillary, also referred to as a keyhole, is formed in which the laser beam can propagate through multiple reflections so that the energy is absorbed along the complete depth of the capillary [4]. The keyhole diameter is approximately one to one and a half times the size of the beam diameter at the material surface [5] and is filled with metal vapor and shielding gas. 
Although the keyhole formation has been well-explained, its dynamic behavior is not fully understood; this is, at least from an experimental point of view, also due to the difficult accessibility of the system. Temperatures exceeding $2000 \mathrm{~K}$ are too high for conventional tactile temperature measurements, and optical accessibility through the keyhole opening is impeded due to the strong emissions caused by the laser-material interaction. The metal vapor, which is partially ionized by the laser beam, can also disturb optical measurements in the vapor capillary, as can the complicated geometrical accessibility due to the small diameter of the keyhole and the fluctuations of the melt pool on short time scales. The temperature of the keyhole wall is assumed to be a parameter of fundamental importance to the keyhole behavior and, thus, to the welding process. As the layer thickness of the melt film, its viscosity, and the surface tension strongly depend on the temperature of the surface material, it is obvious that, in order to predict or even control the dynamics of the keyhole, and accordingly, part of the melt pool dynamics, knowledge of the temperature distribution at the keyhole wall is crucial. The small layer of evaporation at the keyhole wall where the material particles are accelerated from the melt film towards the center of the keyhole contributing to the metallic vapor is known as the Knudsen layer [6]. Previous surveys conducted in the field of the numerical simulation of deep-penetration laser welding have already addressed this quantity. For example, it has been predicted by Ki et al. [7] that the melt surface inside the keyhole is overheated, leading to temperatures far beyond the evaporation temperature of the weld material. The model created by Volpp et al. [8] used to investigate keyhole stability supports this prediction by calculating the temperature at the transition layer between the liquid and gaseous phase as ranging from $3400 \mathrm{~K}$ to $3800 \mathrm{~K}$, thereby significantly exceeding the evaporation temperature of the examined material. According to Fabbro et al. [9], the deep welding process can be divided into different regimes corresponding to the welding velocity $v_{\text {weld }}$. The velocity range below $v_{\text {weld }}=5 \mathrm{~m} / \mathrm{s}$ is referred to as the Rosenthal regime, which is characterized by an almost vertical keyhole with circular cross-sections along the laser beam axis. The temperature is thought to be homogeneously distributed along the contour of each cross-section and, therefore, is only spatially dependent on the keyhole depth. For higher welding speeds, the keyhole is tilted and elongated in the welding direction. The tilt leads to an asymmetric laser absorption at the keyhole wall, causing an inhomogeneous temperature distribution. It is therefore evident that, in the Rosenthal regime, other than for higher welding velocities, the local temperature measurement at a certain depth in the keyhole is representative of the whole circumference. Similar conclusions were drawn by Berger et al. [10], who additionally considered the influence of ambient pressure on the keyhole stability in their studies. Focusing on the regime of cylindrical keyhole shapes, Kroos et al. [11] were able to show in their modeling of keyhole dynamics that, for different metals, the overheating at the Knudsen layer always ranges between $50 \mathrm{~K}$ and $100 \mathrm{~K}$ above the evaporation temperature of the respective materials.

The investigations carried out in the course of this study aim to conduct a direct temperature measurement in the vicinity of the frontal keyhole wall, as depicted in Figure 1. Although the melt pool and the keyhole are not as stable as for higher velocities in the Rosenthal regime, the keyhole's properties concerning geometrical features and temperature distributions are suitable for the performed experiments. For a corresponding set of process parameters, measurements at a depth of $h_{\text {meas }}=4 \mathrm{~mm}$ are conducted in order to estimate the absolute temperature values in the region of interest and to demonstrate the feasibility of the introduced measuring method. 


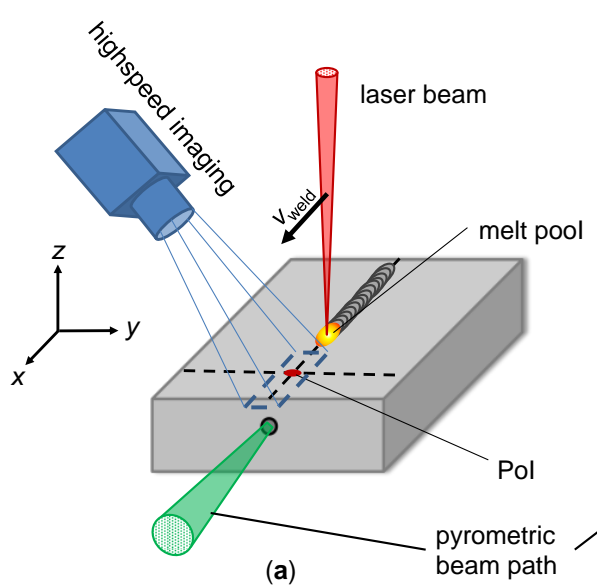

(a)

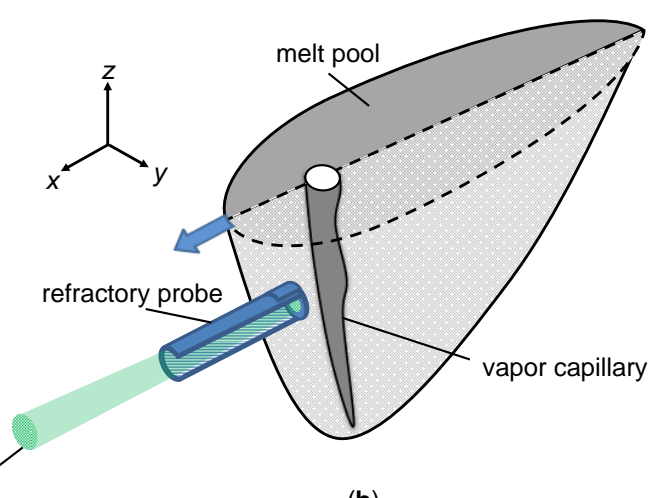

(b)

Figure 1. Schematic of the process observation: (a) total view of the sample showing pyrometry and high-speed imaging methods during the welding process, and (b) close-up on the melt pool probing.

\section{Materials and Methods}

\subsection{Specimen Design}

The specimens used during the experiments consist of two parts. The first part is a block of pure aluminum EN AW-1050A (BIKAR-Metalle GmbH, Bad Berleburg, Germany) with the dimensions $60 \times 40 \times 10 \mathrm{~mm}^{3}$, which acts as the base material for the bead-on-plate welding during the experimental process. This size is chosen as it is big enough to diminish any boundary effects on the temperature propagation on the timescale of the performed measurements. Therefore, on the timescale of interest, the specimen can be treated as a semi-infinite body. Regarding the aluminum and the process laser system used, the intensity threshold for initializing a deep penetration welding process is $I_{\text {key }}=5 \times 10^{6} \mathrm{~W} / \mathrm{cm}^{2}$ according to [12]. The second part of the specimen comprises the refractory probe, which consists of a high-purity tantalum tube (Goodfellow $\mathrm{GmbH}$, Hamburg, Germany) sealed at the front end with 20- $\mu \mathrm{m}$ tantalum foil (Goodfellow $\mathrm{GmbH}$, Hamburg, Germany) using micro deep-drawing, as depicted in Figure 2. With an outer diameter of $d_{\text {out }}=1.05 \mathrm{~mm}$ and an inner diameter of $d_{\text {in }}=0.6 \mathrm{~mm}$, the size of the tube is chosen in order to minimize the disturbance to the melt pool system as much as possible while leaving enough space for the measuring spots of the pyrometers to fit inside completely. The foil is force-fit onto the tantalum tube, establishing only a mechanical connection between the two parts. A drill hole with a diameter slightly bigger than the outer diameter of the probe and with a depth corresponding to the length of the tube $l_{\text {probe }}=8 \mathrm{~mm}$ is drilled into the front side of the aluminum block and equipped with the tantalum tube with the sealed end facing the drill hole, as shown in Figure 3.

This way, a refractory measuring channel is established, maintaining the accessibility of the tantalum foil by the pyrometer throughout the ongoing welding process while shielding it from the prerunning melt. Another important requirement is that the probe maintains its solid state at least during the relevant experimental timespan. The tantalum foil at the end of the probe acts as a temperature screen capable of displaying the temperature values that occur during the welding process in the regions of interest. The temperature of the weld material is projected onto the solid foil, which, according to the adapted temperature, radiates in the manner of a grey body. By maintaining its solid phase, it can be ensured that the emissivity of the tantalum foil does not change abruptly. 


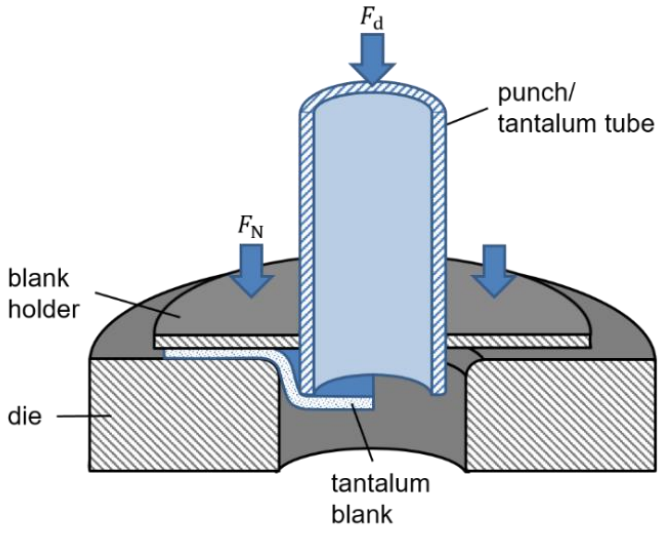

(a)

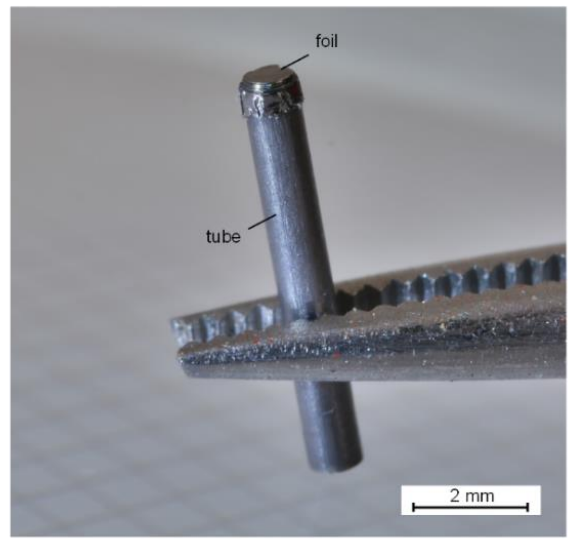

(b)

Figure 2. Preparation of the refractory tantalum probes: (a) deep-drawing process of the tantalum foil, and (b) a finished, ready-to-use probe.

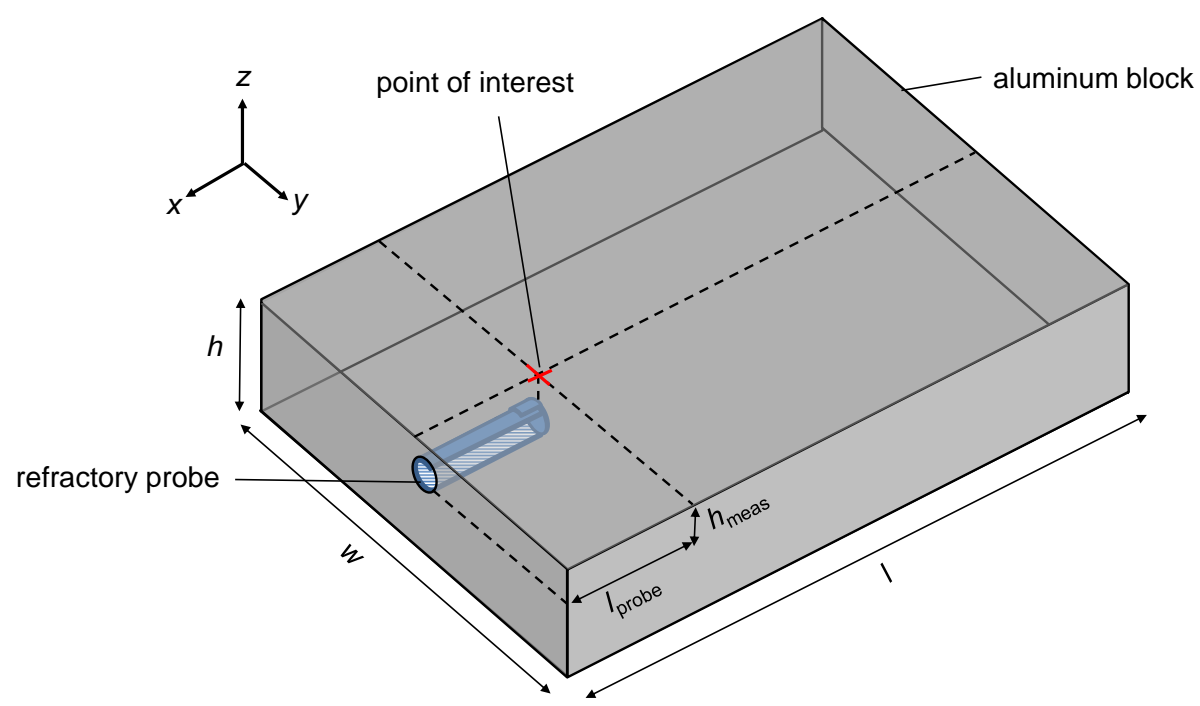

Figure 3. Schematic of the weld sample design with $l=60 \mathrm{~mm}, w=40 \mathrm{~mm}$, and $h=10.5 \mathrm{~mm}$. The length of the probe was set to $l_{\text {probe }}=8 \mathrm{~mm}$, whereas the $z$-position of the probe beneath the sample surface was meant to be varied in order to enable the measurement of a depth-dependent temperature profile. In this setup, the $z$-position of the probe was fixed to $h_{\text {meas }}=4 \mathrm{~mm}$.

\subsection{Experimental Setup}

In the welding process, a TruDisk 12002 disk laser (Trumpf GmbH \& Co. KG, Ditzingen, Germany) operating at a wavelength of $\lambda=1030 \mathrm{~nm}$ with a minimum beam parameter product of $B P P_{\min }=8 \mathrm{~mm} \cdot \mathrm{mrad}$ is used as the laser source. With a core diameter of the optical fiber of $d_{\text {fiber }}=200 \mu \mathrm{m}$ and an image ratio of 3:2, a focal diameter of the laser spot of $d_{\text {focus }}=300 \mu \mathrm{m}$ is achieved theoretically and is verified by a beam profile measurement. The Rayleigh length is given by $z_{\mathrm{R}}=2.52 \mathrm{~mm}$. The welding process is observed by high-speed imaging from the side, as well as by high-speed pyrometry. A schematic view of the experimental setup is presented in Figure 4 . The high-speed imaging is performed using a Phantom VEO 410L (Vision Research, Wayne, NJ, USA) in combination with a Cavilux HF illumination laser (Cavitar Ltd, Tampere, Finland) operating at a wavelength of $\lambda_{\text {ill }}=810 \mathrm{~nm}$ and a narrow band-pass filter with a central wavelength of $\lambda_{\mathrm{BP}}=810 \mathrm{~nm}$ with a full width at the half-maximum of $F W H M=12 \mathrm{~nm}$. This way, the camera can effectively be protected from the scattered radiation from the welding process. The high-speed video is recorded at a framerate of $f_{\mathrm{HS}}=17 \mathrm{kHz}$ and is used for the evaluation of the process stability and keyhole fluctuations, as well as for the localization of the keyhole position relative to the probe. The temperature 
measurement is executed using a KGA 740 (Kleiber Infrared GmbH, Unterwellenborn, Germany), a small-band pyrometer operating at wavelengths in the infrared spectrum at $\lambda_{\text {pyro }}=2000 \mathrm{~nm}$ to $2200 \mathrm{~nm}$ and capable of recording at sampling rates of up to $f_{\text {pyro }}=166 \mathrm{kS} / \mathrm{s}$. The measuring spot has a minimum diameter of $d_{\text {spot }}=0.4 \mathrm{~mm}$ and is focused inside the tantalum tube and onto the tantalum foil at the end of the probe. Thus, the pyrometer records the temperature at a fixed point throughout the process from the beginning until the moment the laser reaches the probe. Due to the laser movement, and consequently, the movement of the melt pool, the relative position between the keyhole and the tantalum foil changes over time at the welding speed. After the destruction of the tantalum foil, the aluminum melt can penetrate the measuring channel so that the measuring spot of the pyrometer moves along with the melt inside the tube. At the same time, the tantalum temperature window is destroyed, and a material transition for the emissivity coefficient changing from the probe material of tantalum to the sheet material of aluminum must be considered when calculating the absolute temperature from the measured voltage signal of the pyrometer. For the validation of the measured temperature signals, additional experiments based on the same set of process parameters are conducted using the H322+ two-color pyrometer (Sensortherm GmbH, Sulzbach, Germany) operating at two discrete wavelengths, namely $\lambda_{1}=1640 \mathrm{~nm}$ and $\lambda_{2}=1400 \mathrm{~nm}$, with a maximum sampling rate of $f_{\text {pyro }}=20 \mathrm{kS} / \mathrm{s}$. The two-color pyrometry is almost independent of the measuring area and always gives values very close to the maximum temperature in the focused region. Even though the temperature can be directly obtained from the quotient of the measured intensities at each wavelength without requiring knowledge of the exact emissivities, it is still reliant on a constant ratio between the emissivity values at each temperature in order to provide reliable information on large temperature intervals. The sampling of the measuring signals is executed with the NI cRIO-9035 FPGA (field programmable gate array) controller (National Instruments, Austin, TX, USA) using the NI-9222 analog input module, allowing for the sampling of four channels with sample rates up to $f_{\text {sample }}=500 \mathrm{kS} / \mathrm{s} \mathrm{simultaneously}$.

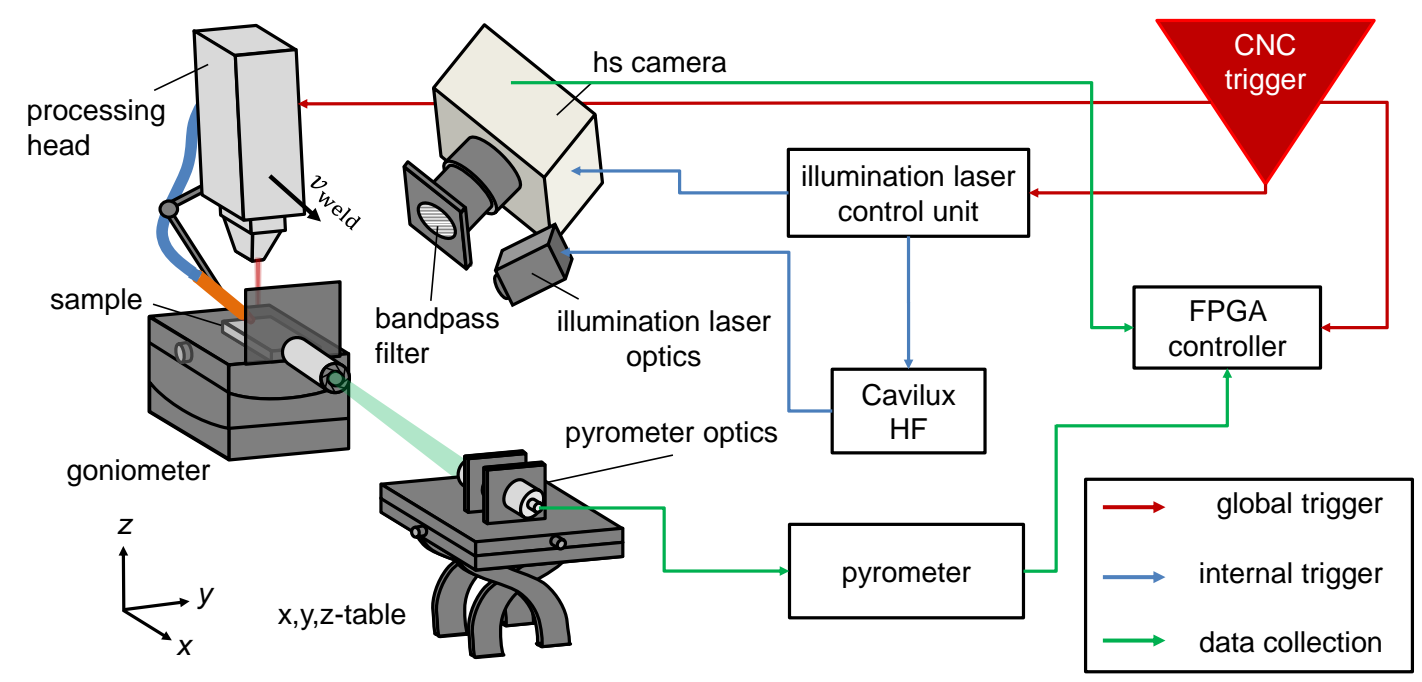

Figure 4. Experimental setup for measuring the temperature near the keyhole front wall. FPGA: field programmable gate array; CNC: computerized numerical control.

\subsection{Measuring Absolute Temperatures with Small-Band Pyrometers over Large Temperature Ranges}

In order to calculate the absolute temperature values from the measured voltage signal received from the small-band pyrometer, the emissivity of the material to be probed must be considered. The emissivity coefficient $\varepsilon$ is not constant but depends on the temperature $T$, as well as on the wavelength $\lambda$ at which the pyrometer is operated. In the most general case, the emissivity depends on both quantities:

$$
\varepsilon=\varepsilon(T, \lambda) .
$$


The emissivity is a factor that determines the share of emitted radiation compared to a black body with an ideal emissivity of $\varepsilon=1$. Considering a measuring spot with an area $A_{\text {pyro }}$ and an effective spectrum $\left[\lambda_{1}, \lambda_{2}\right]$ for the pyrometer, the radiant power to be measured at a given temperature is described by the wavelength integral of Planck's law:

$$
I(T)=\int_{\lambda_{1}}^{\lambda_{2}} \varepsilon(T, \lambda) S(T, \lambda) d \lambda \text { with } S(T, \lambda)=\frac{2 \pi h c}{\lambda^{5}} \frac{1}{e^{\frac{h c}{\lambda k_{\mathrm{B}} T}}-1}
$$

where $h$ describes the Planck constant, $c$ represents the vacuum speed of light, and $k_{\mathrm{B}}$ is the Boltzmann constant.

From Figure 5b, it becomes clear that the analytical approximation of the numerically calculated integral is more accurate for high temperatures. Therefore, the following temperature calculations must be considered more reliable for temperatures exceeding $2000 \mathrm{~K}$, which corresponds to the range of expected keyhole temperatures. As there is no analytical solution for a finite integral of $S(T, \lambda)$, the solution can only be acquired numerically. Considering the emissivity to only have a weak dependency on the wavelength within the given operational spectrum, the radiant power can be calculated numerically for each temperature within the expected temperature range and fitted with a potency function with the fitting parameters $a$ and $b$. It should be noted that this crucial assumption of the spectral independence of the emissivity cannot be assumed in general but only for specific intervals for which such a behavior is documented.

$$
P(T)=A_{\mathrm{pyro}} \cdot I(T) \approx A_{\mathrm{pyro}} \cdot a \cdot T^{b}
$$

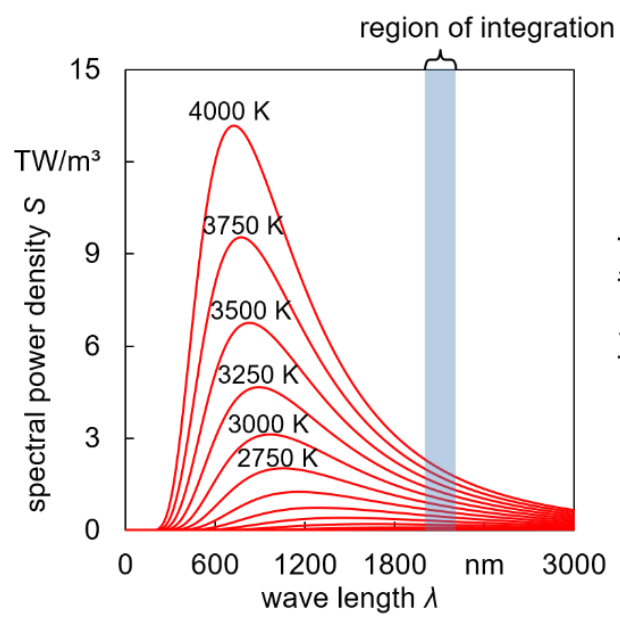

(a)

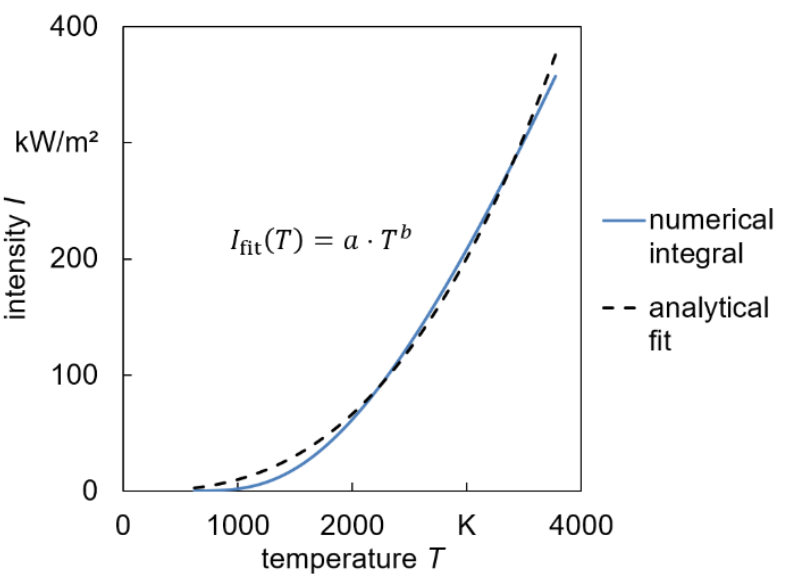

(b)

Figure 5. Theoretical heat-induced radiation: (a) array of curves showing the black body radiation for different temperatures, and (b) corresponding numerical wavelength integral for the operating spectrum of the pyrometer.

As during the experiments, all temperature values are recorded with an emissivity preset of $\varepsilon=1$, it is possible to define a calibration coefficient $\xi$ for the temperature.

$$
\xi(T)=\frac{T(P, \varepsilon)}{T(P, 1)}=\varepsilon(T)^{(-1 / b)}
$$

This calibration coefficient enables the calculation of the absolute temperatures from a noncalibrated

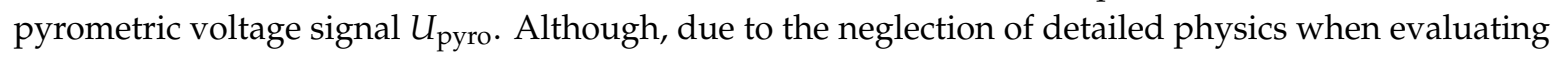
the emissivity, this postmeasurement evaluation of the temperature $T$ cannot be assumed to be as accurate as a material-specific calibration, it can be very helpful in investigating large temperature 
ranges, as well as temperatures that may be too high for sophisticated calibration under conventional laboratory conditions. The Equations (3) and (4) would hold for an ideal pyrometer covering the whole temperature scale starting at $T_{\min }=0 \mathrm{~K}$. Due to technical constraints, the detectable temperatures have a lower and an upper boundary, $T_{\min }$ and $T_{\max }$, each corresponding to temperature values greater than $0 \mathrm{~K}$. In this case, the calibration coefficient only acts upon the temperature difference within the measuring interval $\Delta T=T-T_{\min }$. It proves that, especially in the region of high temperature values as they lie in the scope of this investigation, the form of the analytical approximation given by Equation (3) is also suitable for $\Delta T$.

$$
\begin{aligned}
& P(T)=A_{\text {pyro }} \cdot I(T) \approx A_{\text {pyro }} \cdot \widetilde{a} \cdot \Delta T^{\widetilde{b}} \\
& \widetilde{\xi}(T)=\frac{T(P, \varepsilon)-T_{\min }}{T(P, 1)-T_{\min }}=\varepsilon(T)^{(-1 / \tilde{b})}
\end{aligned}
$$

From Equation (6), it can be seen that the calibration coefficient can be obtained from the temperature difference in an analogous manner. The pyrometric temperature measurement starts at the temperature offset $T_{\min }$, and the calibration coefficient $\widetilde{\xi}(T)$ only acts upon the temperature difference $\Delta T$.

$$
\begin{gathered}
\Delta T(P, 1)=s \cdot U_{\text {pyro }} \text { with } s=\frac{T_{\max }-T_{\min }}{\Delta U} \\
T=\tilde{\xi}(T) \cdot \Delta T(P, 1)+T_{\min }=\widetilde{\xi}(T) \cdot s \cdot U_{\text {pyro }}+T_{\text {min }}
\end{gathered}
$$

When calculating the actual temperature from the pyrometric voltage signal, it becomes evident from Equation (8) that $T$ depends on itself as the emissivity depends on the temperature in an empirical manner. Therefore, the equation cannot be solved for $T$ analytically. Using a self-consistency approach, a solution for $T$ can be found by iterating the self-consistency cycle until the input and the output temperatures match within a defined tolerance $\delta$, as depicted schematically in Figure 6.

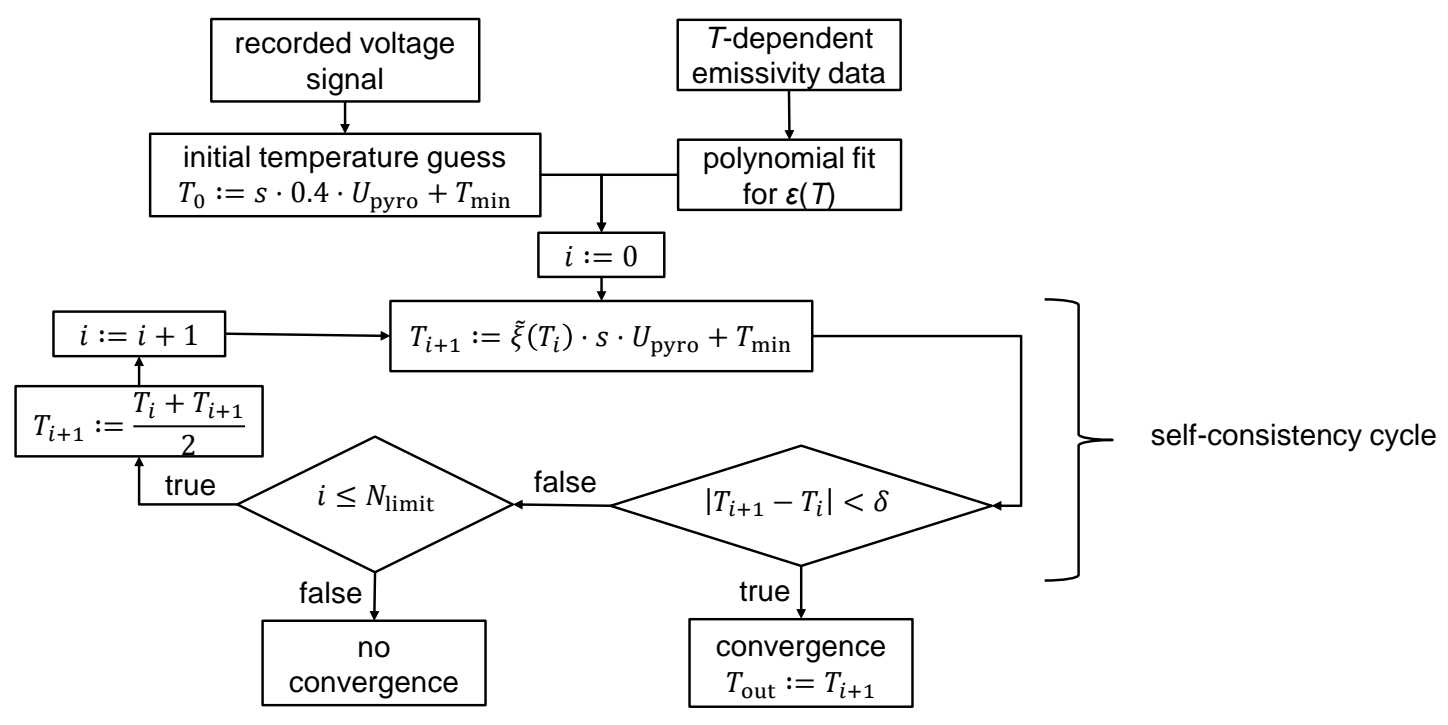

Figure 6. Flow chart of self-consistent temperature calculations.

\subsection{Evaluation of the Robustness of the Pyrometric Measurements}

A major experimental error concerning the temperature measurements arises from the positioning inaccuracy of the measuring spot inside the refractory probe. As both the cross-section of the probe and the measuring spot exhibit a circular shape, the positioning inaccuracy can be defined as a radial shift between the center points of both circles. The error of the measuring spot is consequently calculated as 
the difference between the circular segments of both circles corresponding to their intersection points at $s_{1}$ and $s_{2}$, as shown in Figure 7 a.

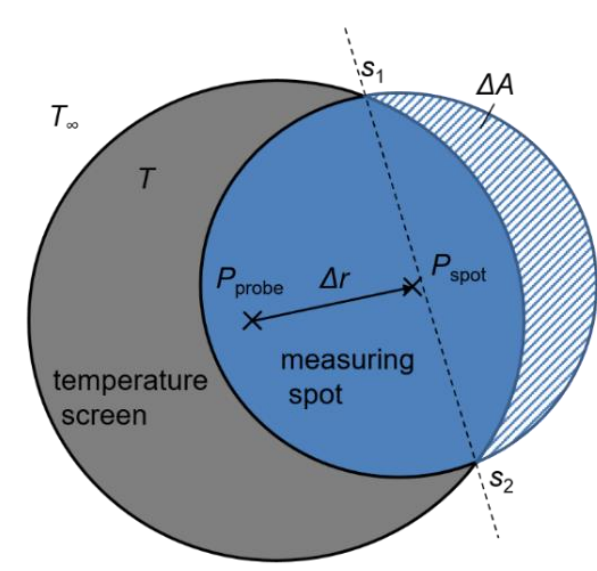

(a)

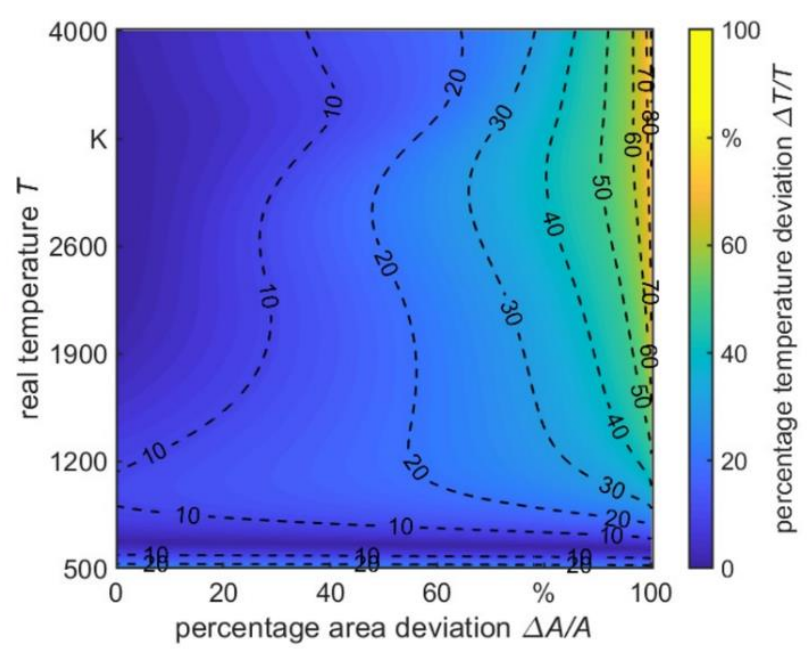

(b)

Figure 7. Influence of positioning errors on the pyrometric measurements: (a) geometric deviation of the measuring spot, and (b) resulting relative temperature deviation exemplarily for the emissivity of tantalum.

For small-band pyrometers, the measured temperature depends strongly on the overlap between the measuring spot and the area of interest. An insufficient overlap has a drastic impact on the outcome of the measurement, as can be seen in Figure $7 \mathrm{~b}$ for the example of the emissivity of tantalum (Ta). It is important to mention that the relative error increases for high temperatures as the deviation between the measured intensities becomes more significant. The expected temperatures occurring in the vicinity and inside the keyhole lie beyond $2000 \mathrm{~K}$. With a positioning error leading to deviations of the measuring area of less than $20 \%$, the reliability of temperature measurements in the region of interest is reasonable.

\subsection{Synchronization of the Measuring Signals}

Regarding the geometrical characteristics of the melt pool generated during deep-penetration laser welding, including the vapor capillary, the different regions that are probed along the welding axis can obviously be of very short extent. The Knudsen layer, with a theoretical thickness in the order of magnitude of $L_{\mathrm{K}} \sim 10 \mu \mathrm{m}$ [13], is the thinnest characteristic keyhole region to be distinguished in the literature. Welding at welding velocities in the range of $30 \mathrm{~mm} / \mathrm{s}$ up to $65 \mathrm{~mm} / \mathrm{s}$ therefore corresponds to very short time intervals of about $t_{\mathrm{K}} \sim 100 \mu \mathrm{s}$ in which the characteristic behaviors can be probed. Due to this temporal restriction, not only are high sampling rates necessary but, also, the synchrony of the employed measuring devices must be assured within strict tolerances. The employment of different measuring devices is always affected by a time delay $\delta t$ between the different signals resulting from the inner runtimes of the respective devices. Therefore, even though the signals are sampled simultaneously by the FPGA controller, they do not necessarily correspond to the same real-time event. Figure 8 shows a schematic of the signal paths from the experimental setup. The internal runtime and signal processing of the high-speed camera and the pyrometer lead to a delay between both signals, preventing a synchronous correlation within the strict tolerances required by the spatial extent of the investigated system. 


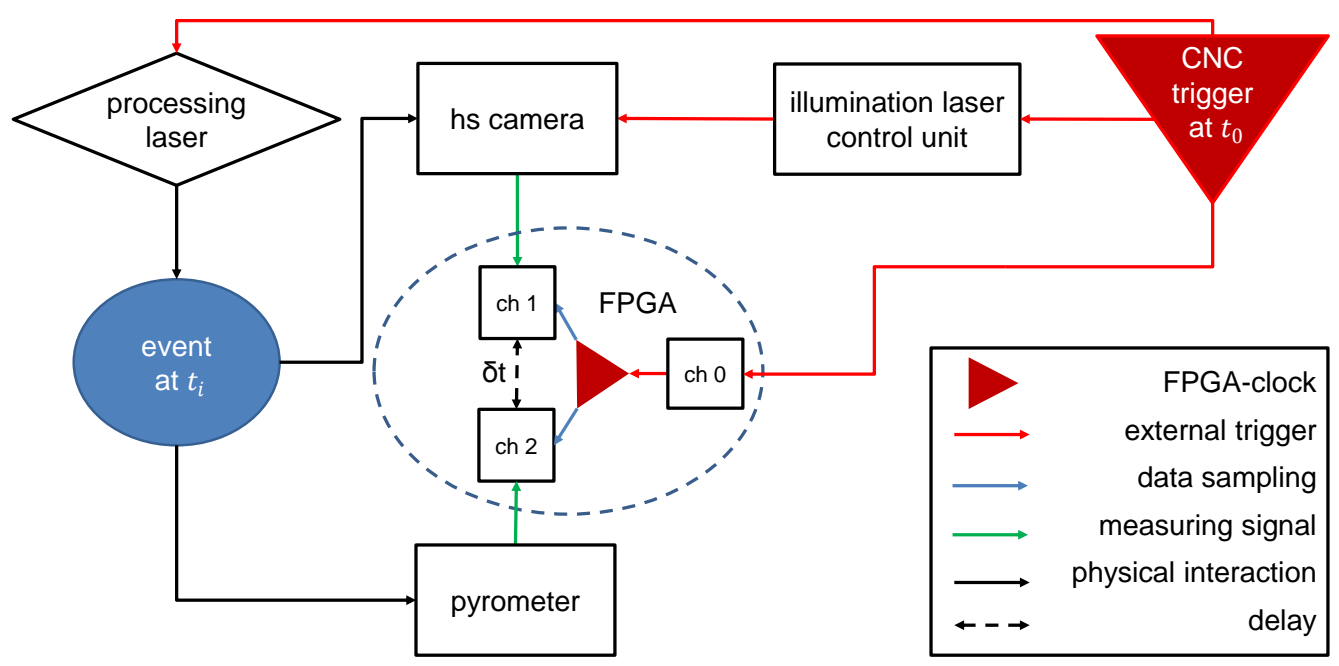

Figure 8. Schematic of the signal paths in the experimental setup.

In order to analyze these internal delays, an experimental setup has been designed in which an event is created that triggers the applied measurements simultaneously during well-defined and short time intervals, as depicted in Figure 9. The continuous heat source that is to be detected by the pyrometer is implemented by a tungsten lamp with an operating temperature of approximately $\mathrm{T}_{\mathrm{W}} \approx 2000{ }^{\circ} \mathrm{C}$. The detectable event for the high-speed camera is given by the visibility of the illumination laser spot. A shutter disk with a radius of $R=47.5 \mathrm{~mm}$ is used to interrupt the corresponding beam paths with a frequency defined by the rotation speed and the diameter of the semicircular notches. The beam path of the illumination laser and the focus of the pyrometer are arranged in such a way that the camera and the pyrometer can detect the event at the same angular position of the shutter disk. Based on the factual synchronicity of the physical events, it is now possible to match the measured signals and thereby find the systematic delay between the measured signals.

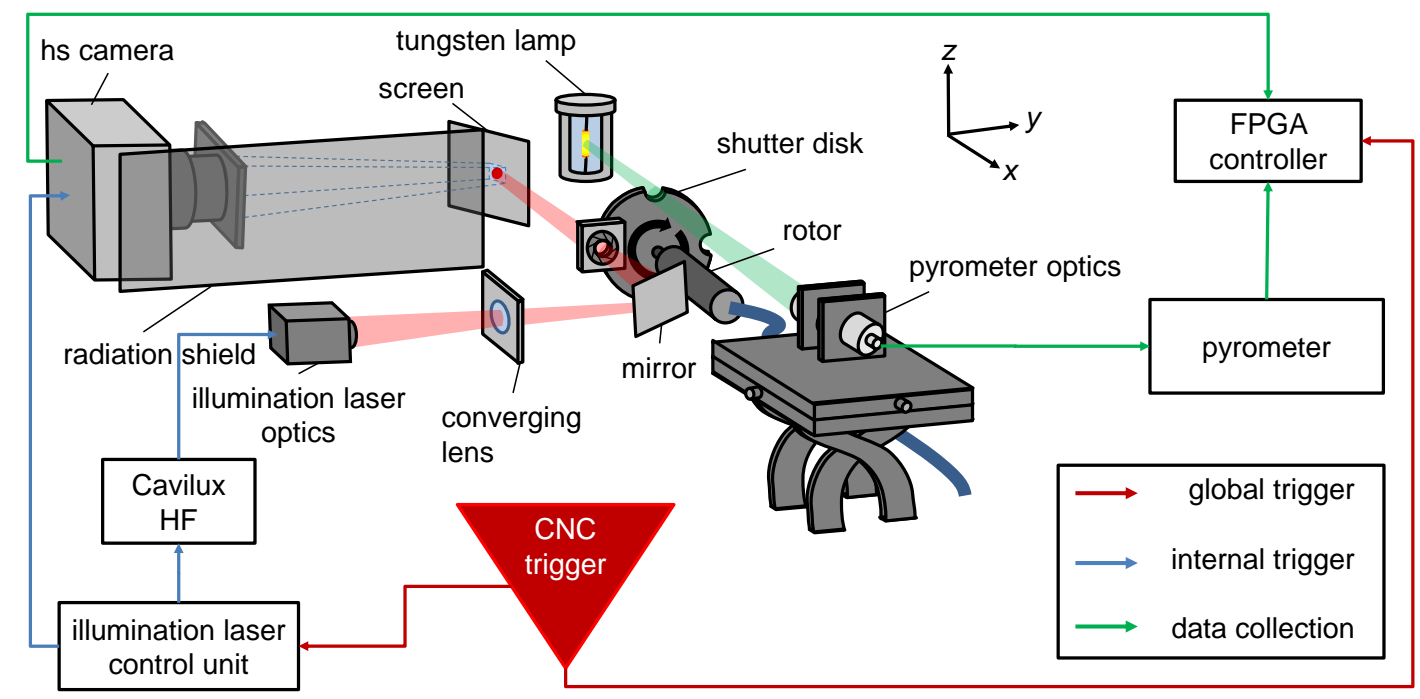

Figure 9. Experimental setup for synchronizing the measuring devices. hs: high-speed.

The accuracy of the synchronization process is calculated from the angular velocity $\omega$ of the shutter disk, the radius of the disk $R$, and the radius of the notches $r$ at the disk rim.

$$
\delta t=\frac{2 \cdot \arctan \left(\frac{r}{R}\right)}{\omega}
$$


The high-speed recording during the synchronization setup is performed at a frame rate of $f_{\mathrm{HS}}=100 \mathrm{kHz}$, matching the adjusted sampling rate of the FPGA controller. The signals from both the pyrometric and high-speed images are postprocessed with threshold values and thereby transformed into binary data indicating the detection of the event or the idle time, respectively. The shift between the recorded signals is analyzed by cross-correlation, showing a significant peak at the configuration of a maximal overlap between the input curves. Given a maximum rotation frequency of $f_{\text {rot }}=330 \mathrm{~Hz}$ according to Equation (9), the maximum precision is given at a temporal uncertainty of $\delta t_{\min }=40 \mu \mathrm{s}$. As this uncertainty is smaller than the exposure time used for high-speed recordings during the experiments of $\Delta t_{\mathrm{HS}}=59 \mu \mathrm{s}$, the setup allows for synchronization between temperature data and high-speed recordings within the accuracy of a single high-speed frame.

\section{Results and Discussion}

The temperature measurement inside the refractory probe was initially conducted with a single parameter set that resulted in a comparatively good quality of the weld seam when welding pure aluminum. The welding velocity was chosen to lie within the range of the Rosenthal regime so that the vapor capillary was expected to have circular cross-sections perpendicular to the laser beam axis with a homogeneous temperature distribution along its contour. The laser was operated at a power of $P=5000 \mathrm{~W}$ with a focus shifted below the workpiece surface, thereby enlarging the keyhole diameter. The defocus was chosen to result in a keyhole diameter on the workpiece surface $d_{\text {key }}$ between $600 \mu \mathrm{m}$ and $900 \mu \mathrm{m}$, based on Skupin [5] and the previously measured beam profile. The welding velocity was set to $v_{\text {weld }}=50 \mathrm{~mm} / \mathrm{s}$, resulting in an energy per unit length of $E_{\text {dist }}=100 \mathrm{~J} / \mathrm{mm}$.

An example temperature curve is depicted in Figure 10. The timespan during which temperature values exceeding the minimum temperature of the pyrometer were obtained is referred to as region of measurement (RoM). For better orientation, the origins of the time and length scales were chosen to coincide with the moment or position, respectively, where the center axis of the laser beam reached the tip of the probe. This convention is used for the presentation of all the following results. Although the curve depicted in Figure 10 shows temperature values from past the keyhole position containing different temperature maxima, as well as a pronounced cooling-down phase, according to the experimental design, only measurements taken from the keyhole prerunning zone can contain reliable information on the temperature. The region past the keyhole cannot be interpreted due to destruction of the probe. Nonetheless, information like the course of the cooling-down of the molten material obtained from the trailing region behind the keyhole can be used for validation of the temperature calculations by indicating whether the temperature curve matches significant points of the phase transitions. Synchronizing the high-speed recordings with the pyrometric measurements, a small region is identified between $t=-25 \mathrm{~ms}$ and $t=-10 \mathrm{~ms}$ where the position of the keyhole front coincides with the position of the tantalum foil, and, therefore, the temperature of the front keyhole should be approximated by the measurement. Inside this short region, the maximum temperatures recorded from different experiments range from $T_{\text {high }}=1415 \mathrm{~K}$ to $2398 \mathrm{~K}$. The fact that the initial slopes of all recorded temperature curves are steep and start at high values close to the melting temperature of the base material is probably due to imperfections in the drill hole geometries. A cross-section polish of the hole shows that, at the bottom, the shape shifts from cylindrical to conical. In the bottom region, the diameter decreases with the increasing depth; thus, the refractory probe cannot establish a laminar contact with the bottom of the drill hole. The small space between the tantalum foil sealing the probe and the bottom of the drill hole traps air, which acts as a thermal isolator. Not enough heat for the foil to emit radiation in the range detectable by the pyrometer is transferred from the weld material until the melt reaches the cavity and starts transferring heat to the foil directly by heat conduction. Therefore, the temperature curves exhibit almost no trace of the prerunning heat. A more problematic effect on the experimental procedure resulting from the entrapped air is that, depending on the volume of air and the stability of the melt pool, the air can expand rapidly, causing a massive blow-out and 
disturbing the measurements completely. In the measurements conducted during this study, such a blow-out at the point of interest occurred in almost $50 \%$ of cases.

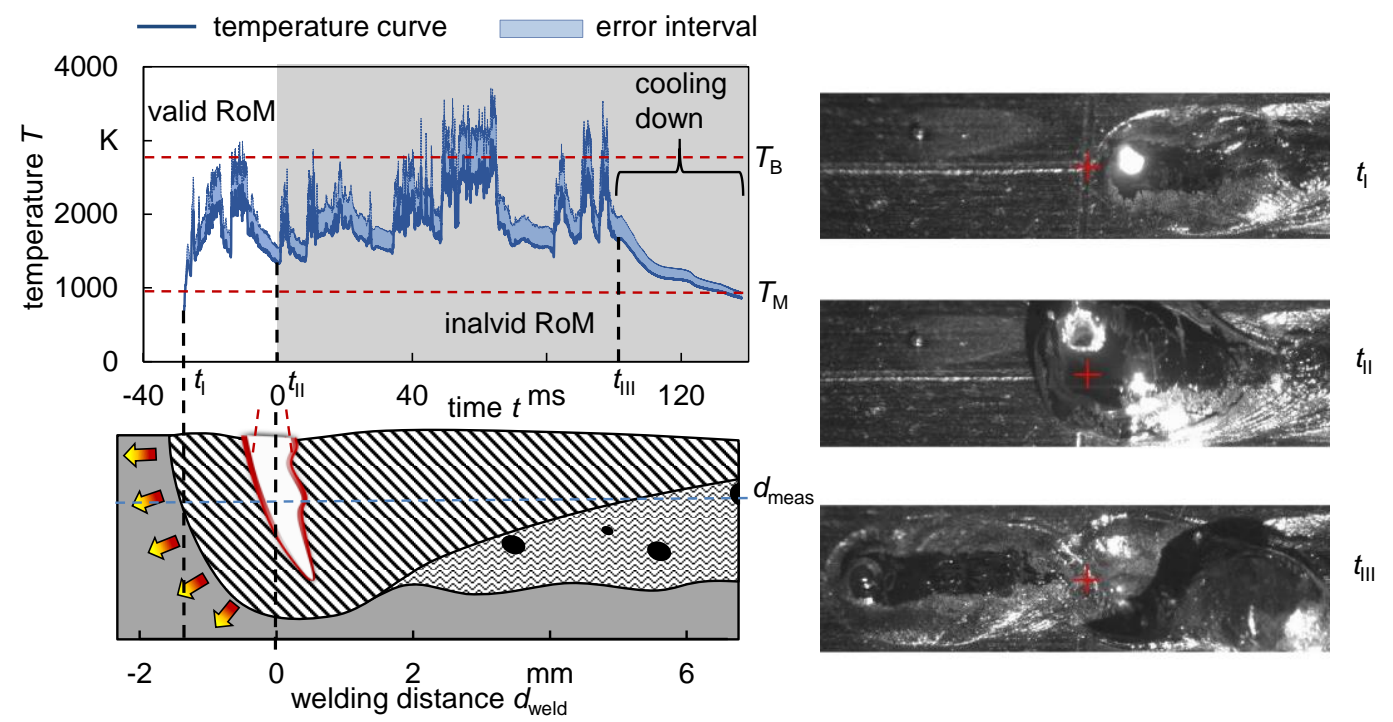

Figure 10. The measured temperature profile with corresponding positions inside the melt pool. RoM: region of measurement.

Besides the issue of imperfections of the sample design, the reproducibility of the experiments is additionally subjected to fluctuations in the shape of the keyhole. Especially the fluctuations of the keyhole depth as depicted in Figure 11 are important to the interpretation of the measured temperatures. These fluctuations are not predictable by now and cannot be controlled by the experimental boundary conditions. Although the absolute measuring depth as seen from the sample's surface is controlled by the sample design, the relative measuring depth at the keyhole wall is also determined by the total keyhole depth itself. As it is not possible to predict or even control the fluctuations during each measurement performed under equal experimental conditions, the relative measuring depth is arbitrary, as the keyhole depth in the valid RoM remains unknown. In Figure 11, it is shown schematically that, at $t_{2}$, the keyhole depth takes the value $l_{\text {key }}\left(t_{2}\right)$. As $l_{\text {key }}(t)$ is also sensitive to boundary conditions that are beyond the control of the experimenters, $l_{\text {key }}\left(t_{2}\right)$ might differ for each measurement.
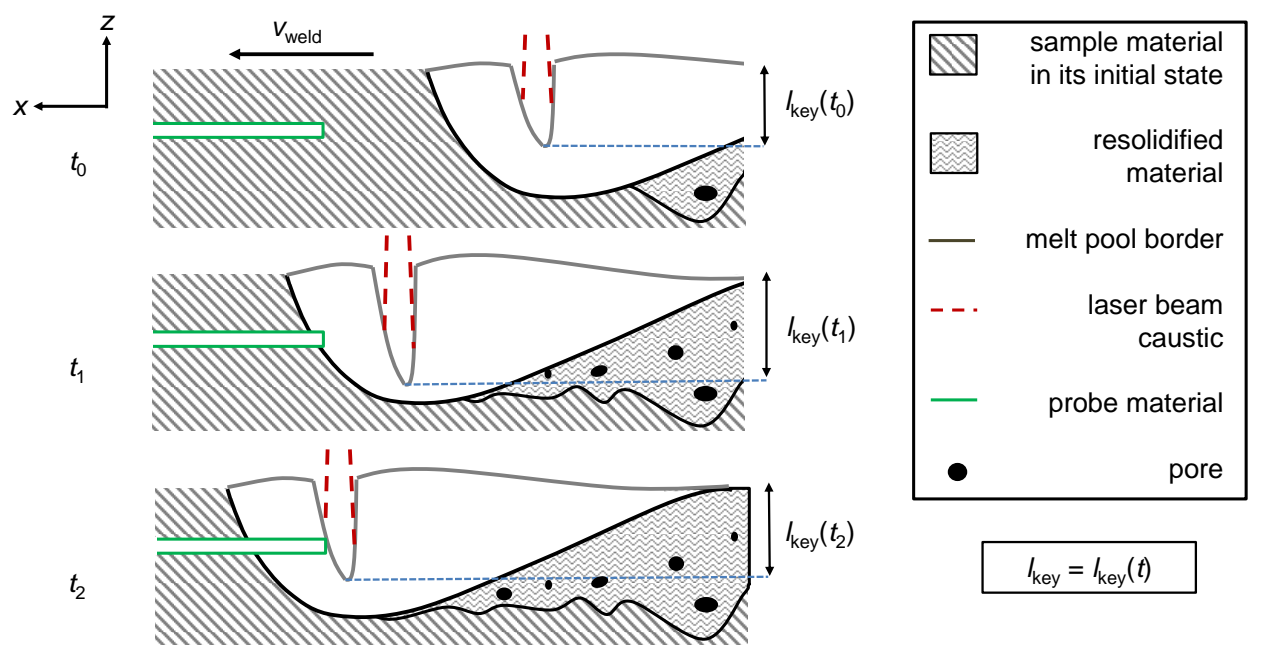

Figure 11. Schematic of the relative position between the probe and the melt pool during different phases of the ongoing measurements. 
Throughout the experiments, eight measurements were conducted successfully. The temperature profile obtained from the valid RoM, as distinguished in the previous section, is chosen as the example for discussion (Figure 12). The temperature starts at values slightly below the melting point of aluminum. In the beginning, the temperature curve is rather smooth and, then, becomes increasingly noisy. In this early stage of the interaction between the melt pool and the refractory probe, damage to the probe is unlikely, so the noise is not ascribed to scattered radiation or reflections of any kind but, rather, to potential vibrations of the melt pool that are transferred to the probe. Thereby, the angle between the temperature screen and the beam path of the pyrometer might change in short time scales, influencing the detected intensity of the thermal radiation.

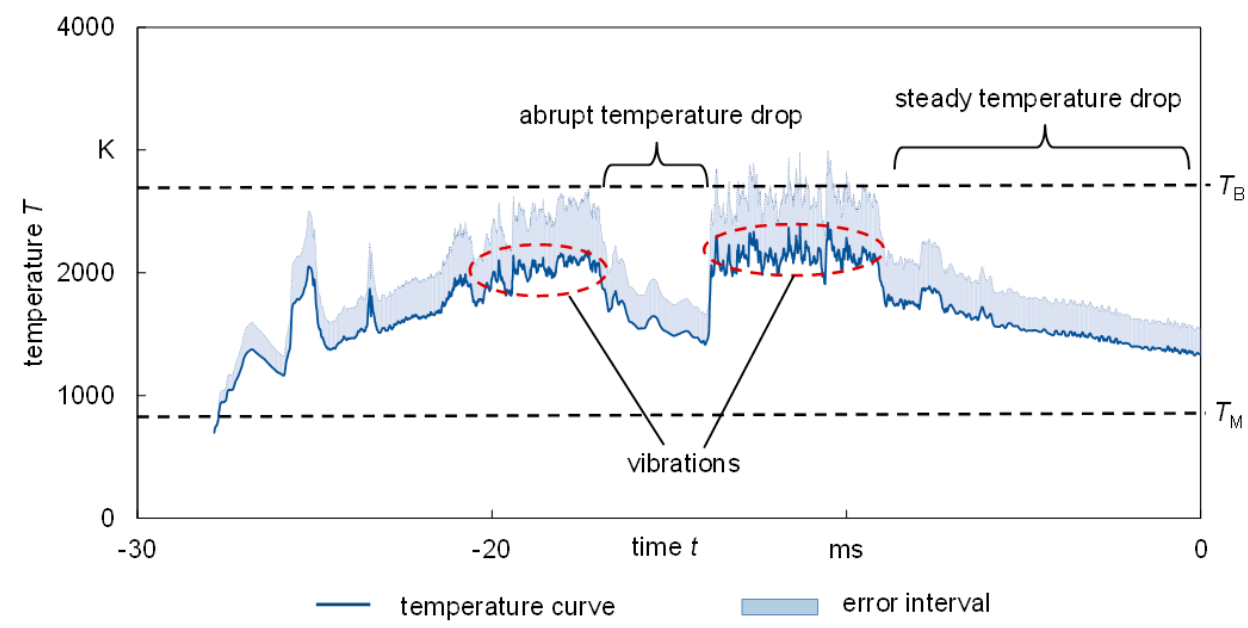

Figure 12. Temperature course from the prerunning melt to the probe destruction, i.e., within the keyhole prerunning region.

The pronounced temperature drop at the end of the depicted region is related to the blow-out that occurs when the keyhole reaches the tantalum foil. It is possible that the fact that the temperature drops within a finite time interval and not abruptly is due to the cooling of the tantalum foil as a consequence of the exposure to gaseous convection by shielding and ambient gas entering the crater left behind by the blow-out. Strong fluctuations of the temperature at time scales of $2 \mathrm{~ms}$ up to $6 \mathrm{~ms}$ can be seen at the beginning at $t \approx-26 \mathrm{~ms}$ and in the middle of the displayed curve at $t \approx-15 \mathrm{~ms}$. To the best of our knowledge, it remains unclear whether these deviations emerge from changes in the probe structure, e.g., from the thermal expansion of the tantalum foil, thereby changing its curvature or its whole position on the tube. This could happen as the force-fit connection between the tantalum foil and the tube may no longer exist when the foil starts to expand at high temperatures. The exposition to the flow velocity of the prerunning melt could cause a tilting of the temperature screen, which could account for the strong and abrupt temperature changes on time scales too long, as they could be ascribed to vibrations. The latter ones, as they are labeled in Figure 12, exhibit frequencies of $f_{\text {vib }}=2 \mathrm{kHz}$ to $5 \mathrm{kHz}$ and, therefore, possibly emerge from the keyhole oscillations, according to [14]. A comparison of the two temperature measurements obtained during the weld processes executed under equal conditions and with the same set of process parameters is given in Figure 13. In both experiments, a blow-out occurred when the keyhole reached the approximate position of the refractory probe; thus, the measurements are comparable judging by the apparent outer circumstances. Although the curves exhibit deviations of up to $\Delta T=800 \mathrm{~K}$ at certain points, qualitatively, they show significant similarities. Apparently, the temperature profile represented by the red curve in Figure 13 shows a rather smooth course in contrast to the second curve. This is an indicator that noisy temperature curves in this region of measuring may indeed be ascribed to an imperfect sample design rather than indicating physical phenomena in the investigated system. 


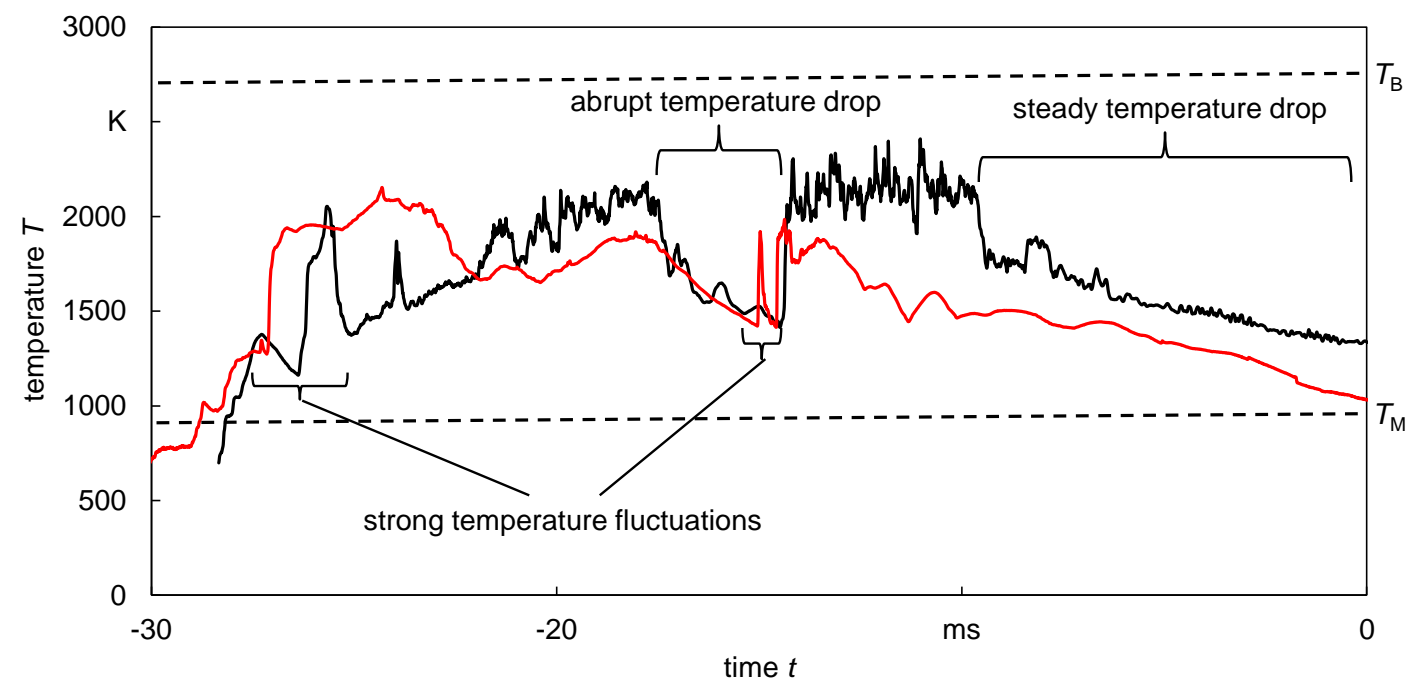

Figure 13. Comparative temperature curves of two independent measurements with the same process parameters.

Considering the estimated size of the keyhole of about $d_{\text {key }} \approx 600 \mu \mathrm{m}$ to $900 \mu \mathrm{m}$ at the top and regarding the typical shape becoming narrower with the increasing depth, the inner probe diameter of $d_{\text {probe }}=600 \mu \mathrm{m}$ is too large to perform a pointwise measurement at the keyhole front, as depicted schematically in Figure 14. Instead, it is likely that the recorded temperatures correspond to the average heat radiation $\bar{I}$ emitted from the tantalum screen. The real temperature of the foil, in turn, results, on the one hand, from the heat transfer mechanisms acting on its outside surface, i.e., of the region of interest within the melt pool in the vicinity of the keyhole front wall, and, on the other hand, from heat propagation inside the tantalum foil. Based on Equation (2) and using the notations as shown in Figure 14, the average intensity on the temperature screen is given by:

$$
\begin{gathered}
\widetilde{T}\left\{\begin{array}{c}
T_{\text {foil }} \forall y, z \in A_{\text {foil }} \\
T_{\text {cross }} \forall y, z \in A_{\text {cross }}
\end{array} \text { and } A_{\text {tot }}=A_{\text {foil }}+A_{\text {cross }}\right. \\
\bar{I}_{\text {pyro }}=\iint_{y, z \in A_{\text {tot }}}\left[\varepsilon(\widetilde{T}(y, z)) \cdot \int_{\lambda_{1}}^{\lambda_{2}} S(\widetilde{T}(y, z), \lambda) d \lambda\right] d y d z
\end{gathered}
$$

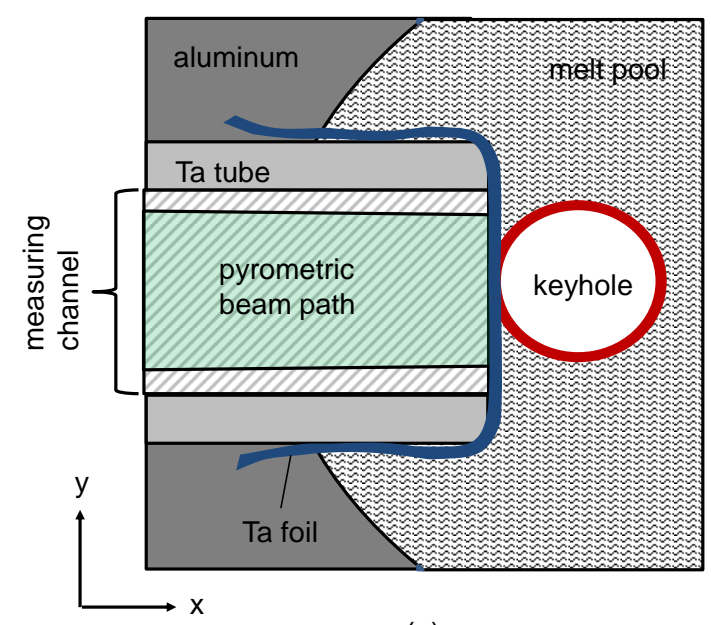

(a)

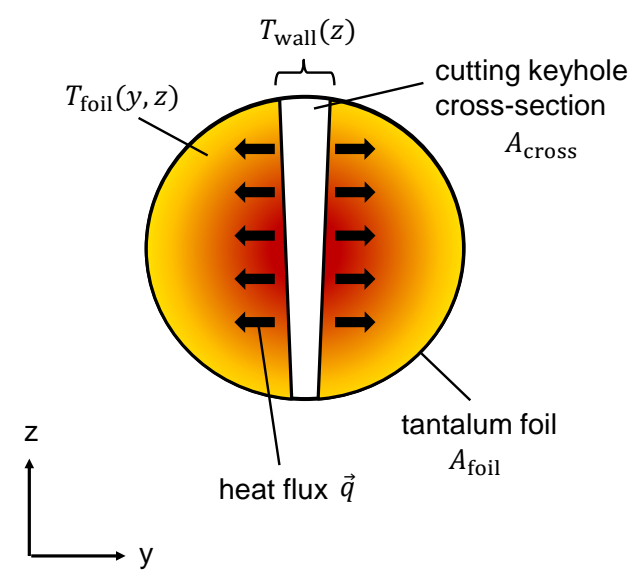

(b)

Figure 14. Geometric relation between keyhole and temperature screen: (a) view along the laser beam axis on the geometric intersection of the keyhole and the Ta foil, and (b) section between the keyhole and the temperature screen as seen by the pyrometer. 
Clearly, as the melt flowing around the keyhole is assumed to be significantly colder than the keyhole itself and contributing to the measured temperature and due to the spatial expanse of the temperature screen, the introduced measuring method inherently underestimates the real temperature at the point of interest.

$$
T\left(\bar{I}_{\text {pyro }}\right)<T(I(y, z)) \forall y, z \in A_{\text {cross }}
$$

Furthermore, due to effects like temperature diffusion on time scales matching the measuring intervals for certain regions, the temporal and spatial resolutions of the measurements in the welding direction are blurred. Under those terms, a color pyrometer is the better choice for measuring the wall temperature.

In a second experimental series for the validation of the obtained results, a Sensortherm H322+ two-color pyrometer was employed, as described in Section 2.3. Hereby, in the valid RoM, as referred to in Figure 10, the temperatures show maximum values of $T_{\text {high }}=3110 \mathrm{~K}$ to $3292 \mathrm{~K}$. Considering that, in color pyrometry, values close to the maximum temperature inside the measuring spot are always obtained and there is no need to know the exact temperature-dependent emissivities, the absolute measured temperatures are more reliable than those obtained using small-band pyrometry. Unfortunately, the lower measuring frequency of the color pyrometer does not allow for detailed changes in the temperature course to be recorded and, furthermore, leads to a temporal uncertainty as intensity is collected over a larger time interval. This temporal uncertainty of the measurement is conterminous with a spatial uncertainty of the temperature measurement in the welding direction. The small scales of the melt pool and keyhole geometries, especially in the frontal part, demand a high spatial resolution of the pyrometer in this direction, which the color pyrometer cannot provide. Taking into consideration the fact that the small-band pyrometry is prone to positioning errors and measures a spatially averaged temperature perpendicular to the welding direction, including colder regions, the results from the two different measuring methods do not necessarily have to be perceived as contradictory. Rather, the measurement performed with the small-band pyrometer gives more qualitative information about the course of the temperature curve, whereas the color pyrometer measures a reliable maximum temperature while lacking sufficient temporal resolution.

\section{Conclusions}

This study presented an experimental setup that enables high-speed temperature measurements within the prerunning melt pool in the vicinity of the keyhole during deep-penetration laser welding. The temperature measurements were supplemented by high-speed videography to identify specific phenomena inside the temperature profile. Based on the conducted experiments, the following conclusions can be drawn:

- Direct in-process temperature measurements at a certain area inside the weld material up to the moment when the keyhole front wall reaches the point of measurement are possible by employing refractory tantalum probes. Up to now, the measuring method affects the process dynamics by causing blow-outs that are ascribed to air inclusions between the tantalum foil and the drill hole bottom.

- The measurements performed with the small-band pyrometer exhibit temperature values lying significantly above the boiling point of the sample material.

- The maximum temperatures in the approximate vicinity of the keyhole wall measured by the small-band pyrometer range between $T_{\text {high }}=2640 \mathrm{~K}$ and $2960 \mathrm{~K}$, thus exceeding the boiling temperature of the base material at its upper boundary by $\Delta T \approx 200 \mathrm{~K}$. Considering these results, an overheating of the keyhole wall, as predicted in the literature, can be confirmed directly. From the discussion, it is concluded that all recorded temperature values obtained within the relevant region only represent a lower temperature boundary at given points in time; thus, the real temperatures are expected to be even higher. 
- Comparative measurements conducted with color pyrometry showed maximum temperatures of up to $T_{\text {high }} \approx 3300 \mathrm{~K}$, supporting the assumption of an overheated keyhole wall, as well as an underestimation of the temperatures recorded by small-band pyrometry.

Author Contributions: Conceptualization, P.W. and R.P.; methodology, R.P. and P.W.; data analysis, R.P.; investigation, R.P.; discussion of contents, R.P. and P.W.; writing-original draft preparation, R.P.; writing-review and editing, P.W.; visualization, R.P.; supervision, P.W.; project administration, P.W.; and funding acquisition, P.W. All authors have read and agreed to the published version of the manuscript.

Funding: This research was funded by the DFG (Deutsche Forschungsgemeinschaft, project number 331150978).

Conflicts of Interest: The authors declare no conflicts of interest.

\section{Abbreviations}

\begin{tabular}{|c|c|c|}
\hline Symbol & Unit & Description \\
\hline$A_{\text {cross }}$ & $\mathrm{mm}^{2}$ & cross-sectional area between the tantalum foil and the vapor capillary \\
\hline$A_{\text {foil }}$ & $\mathrm{mm}^{2}$ & area of the tantalum foil, excluding the cross-sectional area with the vapor capillary \\
\hline$A_{\text {pyro }}$ & $\mathrm{mm}^{2}$ & area of the pyrometric measuring spot \\
\hline$A_{\text {tot }}$ & $\mathrm{mm}^{2}$ & total area of the tantalum foil \\
\hline$B P P_{\min }$ & $\mathrm{mm} \cdot \mathrm{mrad}$ & beam parameter product \\
\hline$d_{\text {fiber }}$ & $\mu \mathrm{m}$ & diameter of the optical fiber \\
\hline$d_{\text {focus }}$ & $\mu \mathrm{m}$ & diameter of the transversal laser beam profile in the focal position \\
\hline$d_{\text {key }}$ & $\mu \mathrm{m}$ & diameter of the keyhole opening \\
\hline$d_{\text {in }}$ & $\mathrm{mm}$ & inner tube diameter of the refractory probe \\
\hline$d_{\text {out }}$ & $\mathrm{mm}$ & outer tube diameter of the refractory probe \\
\hline$d_{\text {spot }}$ & $\mathrm{mm}$ & minimum spot size of the pyrometric measuring beam \\
\hline$d_{\text {weld }}$ & $\mathrm{mm}$ & welding distance traveled by the laser beam \\
\hline$\delta$ & K & convergence threshold for self-consistent temperature calculation \\
\hline$E_{\text {dist }}$ & $\mathrm{J} / \mathrm{mm}$ & energy per unit length \\
\hline$\xi$ & - & temperature calibration coefficient \\
\hline$\varepsilon$ & - & emissivity coefficient \\
\hline$f_{\mathrm{HS}}$ & $\mathrm{kHz}$ & frame rate used for the high-speed recordings \\
\hline$f_{\text {pyro }}$ & $\mathrm{kS} / \mathrm{s}$ & sampling rate of the pyrometer \\
\hline$f_{\text {rot }}$ & $\mathrm{Hz}$ & rotation frequency of the shutter disk used for synchronization \\
\hline$f_{\text {sample }}$ & $\mathrm{kS} / \mathrm{s}$ & sampling rate used by the FPGA controller for data acquisition \\
\hline$f_{\mathrm{vib}}$ & $\mathrm{Hz}$ & vibration frequency of the melt pool or the vapor capillary, respectively \\
\hline FWHM & $\mathrm{nm}$ & $\begin{array}{l}\text { full width at half-maximum of the transmitted wavelength interval of the bandpass } \\
\text { filter }\end{array}$ \\
\hline$h$ & $\mathrm{~mm}$ & height of the weld sample \\
\hline$I$ & $\mathrm{~W} / \mathrm{mm}^{2}$ & intensity \\
\hline$I_{\text {key }}$ & $\mathrm{W} / \mathrm{mm}^{2}$ & threshold intensity of the laser beam required to enable a deep welding process \\
\hline$I_{\text {pyro }}$ & $\mathrm{W} / \mathrm{mm}^{2}$ & $\begin{array}{l}\text { intensity of the heat radiation in the wavelength interval detectable by the } \\
\text { pyrometer }\end{array}$ \\
\hline$l$ & $\mathrm{~mm}$ & length of the weld sample \\
\hline$l_{\text {key }}$ & $\mathrm{mm}$ & length of the keyhole in the direction of the laser beam axis; keyhole depth \\
\hline$L_{\mathrm{K}}$ & $\mu \mathrm{m}$ & thickness of the Knudsen layer \\
\hline$\omega$ & $\mathrm{rad} / \mathrm{s}$ & angular velocity of the shutter disk during the synchronization experiments \\
\hline$P$ & $\mathrm{~W}$ & laser power \\
\hline$q$ & $\mathrm{~W} / \mathrm{mm}^{2}$ & heat flux \\
\hline$R$ & $\mathrm{~mm}$ & radius of the shutter disk used during the synchronization experiments \\
\hline$r$ & $\mathrm{~mm}$ & radius of the notches at the outer rim of the shutter disk \\
\hline$S$ & $\mathrm{~W} / \mathrm{mm}^{3}$ & spectral energy density \\
\hline$s$ & $\mathrm{~K} / \mathrm{V}$ & $\begin{array}{l}\text { straight slope of the translation between pyrometric voltage signal and } \\
\text { temperature difference }\end{array}$ \\
\hline$T$ & K & absolute temperature \\
\hline
\end{tabular}




\begin{tabular}{|c|c|c|}
\hline$T_{\min }, T_{\max }$ & K & minimum/maximum temperature detectable by the pyrometer \\
\hline$T_{\text {low }}, T_{\text {high }}$ & K & minimum/maximum temperature detected during a specific measurement \\
\hline$T_{\mathrm{W}}$ & ${ }^{\circ} \mathrm{C}$ & operating temperature of the tungsten lamp \\
\hline$t$ & $\mathrm{~s}$ & time \\
\hline$t_{\mathrm{K}}$ & $\mu \mathrm{s}$ & $\begin{array}{l}\text { timespan during which temperature values are obtained from inside the Knudsen } \\
\text { layer at a given welding velocity }\end{array}$ \\
\hline$\delta t$ & $\mu \mathrm{s}$ & systematic time delay between the different measuring signals \\
\hline$v_{\text {weld }}$ & $\mathrm{m} / \mathrm{s}$ & relative velocity between the laser beam and the sample surface \\
\hline$\lambda$ & $\mathrm{nm}$ & wavelength of light \\
\hline$\lambda_{\mathrm{BP}}$ & $\mathrm{nm}$ & transmissive wavelength of the bandpass filter \\
\hline$\lambda_{\text {ill }}$ & $\mathrm{nm}$ & operational wavelength of the illumination laser \\
\hline$\lambda_{\text {pyro }}$ & $\mathrm{nm}$ & operational wavelengths of the pyrometer \\
\hline$U_{\text {pyro }}$ & $\mathrm{V}$ & pyrometric voltage signal \\
\hline$w$ & $\mathrm{~mm}$ & width of the weld sample \\
\hline$z_{\mathrm{R}}$ & $\mathrm{mm}$ & Rayleigh length \\
\hline
\end{tabular}

\section{References}

1. Katayama, S.; Kawahito, Y.; Mizutani, M. Latest Progress in Performance and Understanding of Laser Welding. Phys. Procedia 2012, 39, 8-16. [CrossRef]

2. Bliedtner, J.; Müller, H.; Barz, A. Lasermaterialbearbeitung; Carl Hanser Verlag: München, Germany, 2013.

3. Svenungsson, J.; Choquet, I.; Kaplan, A. Laser welding process-A review of keyhole welding modelling. Phys. Procedia 2015, 78, 182-191. [CrossRef]

4. Hügel, H.; Graf, T. Laser in der Fertigung: Strahlquellen, Systeme, Fertigungsverfahren, 2nd ed.; Vieweg + Teubner: Wiesbaden, Germany, 2009.

5. Skupin, J. Nichtlinear Dynamisches Modell Zum Laserstrahlschweissen von Aluminiumlegierungen. Doctoral Thesis, Staats- und Universitätsbibliothek, Bremen, Germany, 2004.

6. Finke, B.R.; Simon, G. On the gas kinetics of laser-induced evaporation of metals. J. Phys. D Appl. Phys. 1990, 23, 67-74. [CrossRef]

7. Ki, H.; Mazumder, J.; Mohanty, P.S. Modeling of laser keyhole welding: Part II. simulation of keyhole evolution, velocity, temperature profile, and experimental verification. Metall. Mat. Trans. A 2002, 33, 1831-1842. [CrossRef]

8. Volpp, J.; Vollertsen, F. Keyhole stability during laser welding-Part I: Modeling and evaluation. Prod. Eng. Res. Dev. 2016, 10, 443-457. [CrossRef]

9. Fabbro, R. Melt pool and keyhole behaviour analysis for deep penetration laser welding. J. Phys. D Appl. Phys. 2010, 43, 445501. [CrossRef]

10. Berger, P.; Hügel, H. Fluid dynamic effects in keyhole welding-An attempt to characterize different regimes. Phys. Procedia 2014, 41, 216-224. [CrossRef]

11. Kroos, J.; Gratzke, U.; Simon, G. Towards a self-consistent model of the keyhole inpenetration laser beam welding. J. Phys. D Appl. Phys. 1993, 26, 474-480. [CrossRef]

12. Matthes, K.-J.; Richter, E. (Eds.) Schweißtechnik: Schweißen von Metallischen Konstruktionswerkstoffen; Mit 130 Tafeln, 3rd ed.; Fachbuchverl. Leipzig im Carl-Hanser-Verl.: München, Germany, 2006.

13. Krivtsun, I.V.; Sukhorukov, S.B.; Sidorets, V.N.; Kovalev, O.B. Modelling of the Processes of Evaporation of Metal and Gas Dynamics of Metal Vapour inside a Keyhole in Laser Welding. Paton Weld. J. 2008, 10, $16-21$.

14. Geiger, M.; Kägeler, C.; Schmidt, M. High-power laser welding of contaminated steel sheets. Prod. Eng. Res. Devel. 2008, 2, 235-240. [CrossRef]

(C) 2020 by the authors. Licensee MDPI, Basel, Switzerland. This article is an open access article distributed under the terms and conditions of the Creative Commons Attribution (CC BY) license (http://creativecommons.org/licenses/by/4.0/). 